Intersections

Canadian Journal of Music

Revue canadienne de musique
Intersections

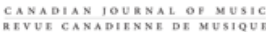

\title{
Le son généreux [pour une hyper-écoute]
}

\section{Chantale Laplante}

Volume 26, numéro 2, 2006

In and Out of the Sound Studio

URI : https://id.erudit.org/iderudit/1013226ar

DOI : https://doi.org/10.7202/1013226ar

Aller au sommaire du numéro

\section{Éditeur(s)}

Canadian University Music Society / Société de musique des universités canadiennes

ISSN

1911-0146 (imprimé)

1918-512X (numérique)

Découvrir la revue

Citer cet article

Laplante, C. (2006). Le son généreux [pour une hyper-écoute]. Intersections, 26(2), 69-74. https://doi.org/10.7202/1013226ar

\section{Résumé de l'article}

L'article que l'artiste propose au lecteur relate le processus de transformation de sa pratique artistique durant une période particulière. L'artiste nous entretient de la transformation dans sa manière de composer la musique et de travailler avec le son. Elle développe sa réflexion autour de deux notions principales : l'hyper écoute qui favorise une approche intuitive, le son devenant la source première d'information à être transmué par la sensibilité de l'artiste, et l'espace-surface issu d'un temps musical non linéaire inscrit dans la durée. Tout au long de l'article, le lecteur prendra connaissance d'un contexte de travail précis qui a favorisé l'émergence d'un questionnement sur le processus de composition musicale et la relation avec le matériau en jeu, le son.
Copyright @ C Canadian University Music Society / Société de musique des universités canadiennes, 2007
Ce document est protégé par la loi sur le droit d'auteur. L'utilisation des services d’Érudit (y compris la reproduction) est assujettie à sa politique d'utilisation que vous pouvez consulter en ligne.

https://apropos.erudit.org/fr/usagers/politique-dutilisation/ 


\section{LE SON GÉNÉREUX [POUR UNE HYPER-ÉCOUTE]}

\section{Chantale Laplante}

Larticle que je propose au lecteur relate le processus de transformation de ma pratique artistique durant une période particulière. La structure d'ensemble de mon texte a conséquemment été commandée par les événements, les rencontres et les lectures qui ont ponctué ce temps fertile. Je suis une praticienne de l'art, et tout comme le peintre Francis Bacon l'affirmait, je crois que "la stimulation et les possibilités logent dans le travail et ne peuvent venir que de là " (Sylvester 1987, 156). Mon texte témoigne ainsi d'une réflexion ancrée dans une mouvance, étoilée de questions et de fragments de pistes, qui alimente la poïétique de mon œuvre.

Tout au long de l'article, le lecteur prendra connaissance d'un contexte de travail précis qui a favorisé lémergence d'un questionnement sur le processus de composition musicale et la relation avec le matériau en jeu, le son. Enfin, une dernière partie a été réservée pour une brève discussion sur la composition dite mixte ${ }^{1}$, dans la mesure où mes travaux en font souvent usage et sont influencés par les interrogations et les explorations discutées ici.

\section{L'ATELIER (LE PIANO)}

Ma résidence d'un an à la "Internationales Künstlerhaus Villa Concordia ${ }^{2}$ a sans nul doute été un élément fondateur d'une série de petits séismes dans ma façon de travailler. Dans le cadre de cette résidence d'artiste, j’avais soudain accès à un temps de création sans préoccupations économiques immédiates, et sans obligations autres que celles face à soi. De plus, j’avais accès à un lieu de travail particulièrement paisible, où logeait un magnifique piano à queue.

Depuis la fin de mes études et l'obtention d'un diplôme de maîtrise en composition instrumentale, je métais lancée corps et âme dans la composition musicale sur commande. De demande de bourse en élaboration d’ouvres, la vie courait devant moi tout autant que les sons. Mon arrivée dans l'espace tranquille d'un nouveau lieu de travail a, comme il arrive souvent dans le contexte de résidences ou de voyages, provoqué des rencontres, des réflexions et des comportements nouveaux.

1 L'CEuvre dite mixte fait appel à la fois aux musiques instrumentale et électroacoustique. La mise en concert impliquera la participation d'un musicien pour jouer la partie instrumentale et la diffusion sur haut-parleurs pour la partie électroacoustique. L’ouvre peut aussi impliquer une manipulation en direct du jeu de l'instrumentiste ainsi qu'une interprétation spatialisée de la partie électroacoustique.

2 La "Internationales Künstlerhaus Villa Concordia » est située à Bamberg en Allemagne. Cette résidence d'artistes fut possible grâce à un programme conjoint ponctuel entre l'État libre de Bavière et le Conseil des Arts et des lettres du Québec, 2002-03. 
Dans le domaine des rencontres, celle avec une œuvre pour clarinette basse et percussions du compositeur Morton Feldman aura eu des répercussions insoupçonnées ${ }^{3}$. Cette pièce tout en piano pianissimo avait un don : celui d'un effet de ralentissement du temps et celui de la nécessité de faire silence. Combien de fois aurai-je écouté cette œuvre? Dès mon arrivée le matin dans l'atelier, je m'enveloppais de cette lente et engageante évocation d'un espace sonore à la fois furtif et saisissant. Sans le savoir, je retournais à l'école : l'école du son... vibrant, prégnant, résonnant d'une matière à la fois diffuse et substantielle.

Au moment de ma résidence, je travaillais à la composition d'une œuvre pour piano, bande et conversation ${ }^{4}$, dont la partie électroacoustique (bande) était à peu près terminée. Toutefois la partie conversation me donnait du fil à retordre (et men donnera encore pendant plusieurs mois) ainsi que la partie piano, celle-ci ayant déjà fait l'objet de deux propositions qui navaient pas données de résultats satisfaisants.

Il était bien difficile de saisir ce piano. Pourquoi? Cela ne pouvait être dû à un manque de connaissance de l'instrument, étant pianiste. Mais peut-être était-ce à cause de cela? Peut-être le temps était-il venu d'entendre cet instrument familier avec des oreilles neuves et un sens plus affiné de son potentiel sonore plutôt que technique? Mais peut-être aussi que les questions soulevées par la composition de cette nouvelle œuvre étaient-elles le reflet de préoccupations plus larges, plus fondamentales, demandant un retournement radical?

En définitive, je reprenais contact non seulement avec le piano comme instrument de musique, mais aussi avec le piano comme instrument de travail. Je constatais que mes outils de simulation sur ordinateur me proposaient un rythme différent, un temps plus agité et je percevais que cela avait pour effet de contredire mon besoin d'une écoute plus lente, plus ressentie. Le travail au piano me donnait la physicalité du geste tactile (les doigts sur la touche) et semblait favoriser l'effort convergent; le temps des gestes peu à peu ouvrait des fenêtres sur un autre temps, imprécis, fugace, propre au domaine de la sensation.

De manière imprévue, le travail au piano prenait un sens qui allait au-delà du fait de composer une musique pour le piano. Au fil des jours, lélaboration de schémas formels s'est faite plus rare, moins nécessaire, pour en venir à constituer un embarras et finalement un barrage. Un barrage à la nécessité de globalement examiner mon attitude avec la composition musicale, instrumentale tout autant quélectroacoustique, et plus particulièrement avec le son. Le piano au centre de latelier maura donné lélan nécessaire pour entrer dans de nouveaux territoires et me diriger vers un nouveau mode découte et de composition musicale, extrêmement attentif et investi de patience.

3 Bass Clarinet and Percussion pour clarinette basse, cymbales, gongs. 1981, 17 minutes.

4 For Piano, Tape and Conversation. [10 minutes]. Commande du Center for Contemporary Arts de Glasgow avec le soutien du Conseil des arts et des lettres du Québec. Première audition : octobre 2002, Free RadiCCals Series, CCA, Glasgow/Lynda Cochrane, piano. 


\section{L'HYPER-ÉCOUTE (LA SENSATION)}

Lespace tranquille. Le piano au centre. Et moi assise au piano. Le piano-son. Le moi-sensation. Un duo central. Centré. Concentré. J'oriente l'oreille vers l'intérieur, dans la sensation au creux du temps tranquille, qui se mélange aux touches. Sans plan préconçu, c'est le plongeon dans l'inconnu. En compagnie du son. Si on veut bien l'écouter. Si on est en mesure de l'écouter bien. Mon approche de la composition musicale se transforme du fait du changement dans la disposition extérieure (l'atelier, le piano) et intérieure (accéder au son par le biais de la sensation). Le son peut-il me suggérer le déroulement de l'œuvre en train de se faire? "L'œuvre d'art est un être de sensation, et rien d'autre : elle existe en soi. » (Deleuze et Guattari 1991, 155).

Je me demande maintenant comme procéder pour faire passer le son dans la sensation et la sensation dans le son. Suis-je encore à la recherche d'une formule? Puis-je accepter ce non-savoir? Ce non-prévoir? Je suis maintenant engagée dans l'espace-intuition, sans modèle, sans prévisions, sans temps.

Lordinateur est maintenant installé sur le piano. La partie bande défile, diffuse. La sensation se précise et les sons apparaissent, résonnent ou non, demeurent ou séloignent. J'observe, j'examine la matière-objet son, et je fais des choix. Mes sons-piano conversent avec ceux de la bande, un trajet se précise, je suis la piste. Je comprends mieux ce que disait Cézanne à propos de l'artiste : "Toute sa volonté doit être silence. Il doit faire taire en lui toutes les voix des préjugés, oublier, oublier, faire silence, être un écho parfait " (Doran 1978, 109). La complexité ne réside pas dans lécriture, mais dans le processus de captation d'un désir intuitif, pour rendre compte, pour permettre, que se déploie la complexité de ma propre sensibilité. Je m’approche de lespace du son dépouillé, dans le sens fondamental d' " ôter ce qui couvre ». Mon écoute du matériau-son s'affine, et permet l'émergence d'un espace sonore plus dilaté.

Le temps tranquille, le retour au piano, la musique de Morton Feldman, un effet de ralentissement, la composition de la partie piano d'une nouvelle œuvre musicale, furent autant d'éléments qui ont permis d'atteindre un nouveau niveau de compréhension de mon travail artistique en questionnant mon attitude et en installant une écoute extrêmement attentive, où chaque note jouée est matièreson, un lieu ouvert et généreux.

L'hyper-écoute représente cette écoute attentive, d'une grande intensité et d'une grande exigence. Il ne s'agit plus d'un do ou d'un $f a$, mais de qualités sonores spécifiques, d'une résonance particulière dans ce registre particulier, qui moffrent peut-être ce que je recherche. Une des difficultés de cette approche est de conserver cet état de concentration et ce lien avec l'intention artistique qui se manifeste peu à peu à travers le son. Le son et moi sommes dans un état d'oscillation, à la recherche de cet "équilibre précaire entre le matériau et sa manipulation " (Feldman 1997, 14-15).

Lobservation implique ici une appropriation et une perte. L'appropriation du son à travers l'hyper-écoute et à travers la perte de la notion du temps; le temps de l'observation et de l'écoute se transforme en celui de la durée : l'espace du son. La perte de la notion du temps fait place à la notion d'espace. Je ne peux percevoir la durée du son que dans la mesure où je lui laisse l'espace: «Le son 
ne doit pas moins être tenu dans son extinction que dans sa production et son développement. " (Deleuze et Guattari 1991, 155). Il faut convoquer ici l'intelligence artistique, qui apprend à tenir compte et à se saisir de tout ce que le son offre dès le moment de son avènement, et dans ce qui le caractérise en intensité, en possibilités directionnelles. Cette attention rebondit dans l'espace de l'auditeur qui éprouvera à son tour et à sa manière ce son que je veux accessible, dans le sens douvert tout à la fois à la question et à la sensation, et à une nouvelle compréhension.

L'hyper-écoute c'est le temps de la patience et d'un formidable silence, qui permet de laisser venir le son, de le laisser être. Le son est à la fois lobservatoire, le lieu de l'observation, et l'objet observé. Il est aussi le tremplin et le lieu d'arrivée de ma sensibilité, qui me permet d’ordonner mon "nuage de sensations $\|^{5}$

\section{L'ESPACE-SURFACE}

C'est dans le déploiement sonore de la durée que peut s'exprimer l'équilibre précaire nécessaire à lélaboration de l’œuvre, ce mouvement de va-et-vient entre l'expression du matériau et la capacité à s'en saisir. Je suis dans la réalité du son dans lequel je me perds et y transmet ma sensation. C'est en apprivoisant ce processus d'aller-retour, en en faisant l'expérience chaque jour dans l'espace tranquille de l'atelier, que s'est amorcé pour moi la conquête d'un espace purement musical, sans linéarité.

C'est dans la pratique intuitive de l'hyper-écoute que j’ai finalement compris la notion de surface-temps telle qu'investiguée par Feldman. " Le temps doit être laissé tranquille " $(1997,207)$. La reconnaissance du son dans sa durée permet le déploiement d'un espace-temps en " surface-espace " dans laquelle l'oreille peut se promener : la surface auditive plane de la musique. Je considère le son pour ce qu'il est, avec sa réalité acoustique caractéristique, et ce faisant, j’accepte que s'imprime en moi cette réalité sonore. La liberté d'expression que je donne au son est aussi celle que je me donne. Il y a là la possibilité d'un abandon, et lélargissement d'une disponibilité pour habiter son espace-intime par la médiation d’un espace-son sans début ni fin.

\section{L'INCLUSION}

L'expérience de l'hyper-écoute développée lors de la composition de la partie piano de la pièce For Piano, Tape and Conversation a aussi eu pour conséquence de dynamiser la relation avec le mode de composition électroacoustique, dans le cadre d'œuvres dites mixtes.

Lorsque le son est placé au centre des préoccupations artistiques, tous les modes de composition ou même de travail " sonore " se rejoignent et interpellent, à la fois chez le créateur et chez l'auditeur, une écoute de type phénoménologique : le son n'est plus un objet interprété, il devient un objet externe qui

5 Expression de Francis Bacon «In working you are really following this kind of cloud of sensation in yourself [...]. " (Sylvester 1987, 149). 
possède sa propre réalité spatio-temporelle ${ }^{6}$. Je crois que mon approche de la composition et plus particulièrement du matériau, qu'il soit de nature acoustique ou électro-concret, s'est en quelque sorte élargie et me permet d'intégrer des processus différents mais ayant en commun un élément fondamental : le son.

L'identification des caractéristiques propres à chaque mode de composition permet de procéder à lénonciation déléments qui interagissent de manière organique. Au foisonnement des sons générés lors du processus de composition électroacoustique répond l'attitude de recueillement de la composition instrumentale. Au son acoustique référencé s'ajoute l'ouverture nouvelle au son inouï de la musique électroacoustique. L'accès à la manipulation des sons et à une production d'informations sonores immense, présente le danger d'un éloignement de l'intention musicale mais tout à la fois multiplie les possibilités d'une ouverture sur une direction sous-entendue.

Dans le contexte de la composition d'une œuvre mixte, je réalise aussi que la question de léquilibre précaire est ici convoqué à la puissance deux: à léquilibre précaire entre le son et sa manipulation, s'ajoute celui de la combinaison de deux mediums différents.

\section{CoNCLUSION}

La démarche effectuée pendant la composition de la pièce For Piano, Tape and Conversation, ma permis de faire certaines observations et de développer une nouvelle manière de vivre le processus de composition.

Mon plus grand étonnement, face à ma relation nouvelle au son, est que cette expérience se soit déroulée dans le contexte de la composition instrumentale. Peut-être n'est-ce pas là un hasard, puisque c'est par et avec le piano que j'ai établi mon premier lien avec la musique, et avec le son. Il est aussi intéressant de noter que ce « retour " au piano s'est effectué dans un contexte d'une tranquillité particulière et d'une intensité ininterrompue propre au contexte d'une résidence d'artistes d'un an. Je comprends aujourd'hui qu'il fallait beaucoup de silence pour que soit possible l'expérience de l'hyper-écoute, où la relation au matériau sonore s'articule dans un mouvement de va-et-vient entre l'intention musicale et le son interpellé et donné : le son généreux.

Le temps du silence, de la patience et de l'hyper-écoute est comme la sousœuvre de la pièce For Piano, Tape and Conversation. Je crois que l'ensemble de la démarche et la combinaison deéléments spécifiques ont participé de manière dynamique à l'émergence d'une musique beaucoup plus personnelle, engagée à un niveau plus profond. Le lieu de la sensation est celui du risque, d'un mouvement intuitif. L'ouvre musicale est un mélange de connaissances techniques et d'une innombrable quantité d'incertitudes. For Piano, Tape and Conversation est le résultat d'une expérience à la fois unique et répétée, dans le sens d'une ré-expérimentation d'un ébranlement vers une essence, du son et ultimement de lêtre.

La difficulté de la maitrise de l'expérience se pose avec la même acuité dans les deux modes de composition, instrumentale et électroacoustique. Les moyens

6 Lire à ce propos la réflexion de Pierre Schaeffer sur la notion d'écoute réduite dans le Traité des objets musicaux. 
sont différents, mais le but est le même : saisir la sensation du matériau, le projeter dans l'espace. Il me faut désormais revivre l'expérience à toutes les échelles, dans une variété de contextes, pour non pas avoir un accès plus rapide au son, mais une réponse plus juste aux questions posées par le son.

Je vise aujourd'hui à dégager les facteurs qui pourraient aider à préciser mon approche et à développer des stratégies adaptées à mes différentes pratiques incluant celle de l'improvisation.

Pour la suite, et comme Giacometti qui dit ne savoir ce qu'il voit qu'en travaillant, je continuerai de ne savoir ce que jentends qu'en travaillant.

\section{BIBLIOGRAPHIE}

Deleuze, Gilles et Félix Guattari. 1991. Qu'est-ce que la philosophie? Paris: Minuit, coll. Critique.

Doran, P.-M. 1978. Conversations avec Cézanne. Paris: Macula.

Feldman, Morton. 1997. Écrits et Paroles, précédés d'une Monographie par Jean-Yves Bosseur. Paris: L'Harmattan, coll. Musique et musicologie.

Schaeffer, Pierre. 1966. Le Traité des objets musicaux. Paris : Éditions du Seuil.

Sylvester, David. 1987. Interviews with Francis Bacon. 2003. Reprint. New York: Thames and Hudson.

\section{RÉSUMÉ}

Larticle que l'artiste propose au lecteur relate le processus de transformation de sa pratique artistique durant une période particulière. L'artiste nous entretient de la transformation dans sa manière de composer la musique et de travailler avec le son. Elle développe sa réflexion autour de deux notions principales: l'hyper écoute qui favorise une approche intuitive, le son devenant la source première d'information à être transmué par la sensibilité de l'artiste, et l'espacesurface issu d'un temps musical non linéaire inscrit dans la durée. Tout au long de l'article, le lecteur prendra connaissance d'un contexte de travail précis qui a favorisé l'émergence d'un questionnement sur le processus de composition musicale et la relation avec le matériau en jeu, le son.

\section{Abstract}

This article deals with a particular period of artistic transformation experienced by the author. The artist describes the transformation that took place in the way she composes music and works with sound. Her reflections are developed around two key ideas: that of hyper-listening, which favors an intuitive approach since sound as a first source of information is transmitted by the sensibility of the artist, and that of space-surface, stemming from a non-linear sense of musical time marked through duration. During the course of the article, the reader will become aware of a specific context of working which promoted an inquiry into the musical compositional process and its relationship with sound, the material at the heart of this process. 